\title{
A Kinetic Study of the Emerging of Grains and Block of Grains from the Inner Volume to the Free Surface of a Cd-Zn Alloy Superplastically Deformed
}

\author{
J. A. Montemayor-Aldrete, ${ }^{1}$ R. Mendez-Olvera, ${ }^{1}$ P. Ugalde-Velez, ${ }^{2}$ \\ G. Torres-Villaseñor, ${ }^{3}$ and $M$. del Castillo-Mussot ${ }^{1}$ \\ ${ }^{1}$ Departamento de Estado Solido, Instituto de Física, Universidad Nacional Autónoma de México, Apartado Postal 20-364, \\ 01000 Mexico City, DF, Mexico \\ ${ }^{2}$ Departamento de Materiales (CBI), Universidad Autónoma Metropolitana-Azcapotzalco, Avenida San Pablo 180, Col. Reynosa, \\ 02200 Mexico City, DF, Mexico \\ ${ }^{3}$ Instituto de Investigación en Materiales, Universidad Nacional Autónoma de México, Apartado Postal 70-360, \\ 04510 Mexico City, DF, Mexico
}

Correspondence should be addressed to J. A. Montemayor-Aldrete, jamonal@fisica.unam.mx

Received 28 September 2012; Accepted 7 November 2012

Academic Editors: R. Hebert and P. Lukac

Copyright (C) 2012 J. A. Montemayor-Aldrete et al. This is an open access article distributed under the Creative Commons Attribution License, which permits unrestricted use, distribution, and reproduction in any medium, provided the original work is properly cited.

\begin{abstract}
A local measurement technique for the study of the kinetic processes of emerging of grains or blocks of grains from the inner volume to the free surface of superplastic materials during deformation is presented and used for the case of the Cd-Zn eutectic alloy deformed at room temperature. This technique could be used to evaluate the approximate time of fracture due to fissure or cavitation growth in samples under superplastic deformation. In principle, this technique will be useful for the development of physical procedures, which allows retarding the process of formation of low mismatch angle, $\bar{\theta}$, between neighboring grains, process which gives place to blocks of grains which dynamically behave as units under the shear stress action. For materials with nanocrystalline structures, such processes are expected to be higher than those of the case of microcrystalline materials.
\end{abstract}

\section{Introduction}

Superplasticity is the ability of polycrystalline material to exhibit, in a generally isotropic way, very high tensile elongations prior to failure [1]. Such phenomena occur mainly by grain-boundary sliding (GBS), between individual grains, with size lower than 10 micrometers, which glide relative to each other with little or no change in shape during long plastic deformation [2, 3]. Also, there exists some experimental evidence for cooperative grain-boundary sliding during superplastic deformation [4-7]. Most of the early studies dealt with microduplex metallic alloys which make the grain growth very difficult, and the unabated scientific interest on superplasticity has from long ago broadened the scope to include intermetallic compounds and nanocrystalline materials [8]. This interest has arisen partly from a scientific viewpoint and partly from the increasing awareness that superplastic materials can be utilized in forming complex shapes in simple and inexpensive forming operations. There are two main advantages in utilizing superplastic materials for metal forming operations. First, large strains can be achieved without necking. Second, the stresses required for superplastic deformation are generally low [9]. Recently, a model which was validated against experimental observations concerning metals, alloys and ceramics of micrometer and submicrometer grain sizes, nanostructured materials, and intermetallics has been proposed to account for optimal superplasticity; their central assumption is that the rate controlling deformation process on GBS is confined to high-angle grain/interphase boundary regions that are essential for grain-boundary sliding developing to a mesoscopic scale (defined to be of the order of a grain 
diameter or more) and for superplastic flow setting in [10]. There are numerous reports of cavity nucleation and growth at grain boundaries during superplastic flow in several different alloys, and some experimental data in $\mathrm{Zn}-\mathrm{Al}$ alloy shows that the cavities are initially reasonably spherical, but they become elongated when the sample pulls out to achieve the maximum superplastic elongation [11]; there are also experimental data which states that superplastic deformation is usually limited by the development of voids or cavities which are seen to form at triple point grain boundaries during the process of GBS [12]. And also there exists direct correlation between the number of cavities nucleated per unit volume and the cumulative average GBS displacement which provides experimental evidence that GBS is the driving force for creep cavity nucleation [13].

It is well known $[14,15]$ that superplastic flow of a material is often described by a constitutive equation of the form

$$
\dot{\epsilon}=\frac{A D G b}{k T}\left(\frac{b}{d}\right)^{p}\left(\frac{\sigma}{G}\right)^{n},
$$

where $\dot{\epsilon}$ is the deformation rate, $D$ is the coefficient of grainboundary diffusion, $G$ is the shear modulus, $b$ is the modulus of Burgers vector, $k$ is the Boltzmann constant, $T$ is the test temperature in Kelvin degrees, $d$ is the average grain size involved in the grain-boundary sliding processes, $p$ is an exponent depending on the grain size (commonly equal to 2), $\sigma$ is the flow stress, $n$ is a parameter inverse to the coefficient of strain rate sensitivity of the flow stress, and $A$ is a constant.

The main purpose of this paper is twofold: (1) to presents a new scanning electron microscopy technique which is applied for the case of the Cd-17.4\% $\mathrm{Zn}$ alloy to measure the kinetically evolution of the emerging of grains or blocks of grains from the inner volume to the free surface of superplastic material during deformation and (2) to develop a simple thermodynamical model about the time evolution of block size of sliding grains during superplastic deformation in order to give some explanation about our experimental data.

\section{Model}

It is well known that there are some general thermodynamical properties of grain boundaries in polycrystalline solids which are relevant to the superplasticity phenomenon: (a) the grain-boundary surface energy, $\gamma(\theta)$, is an approximately linear increasing function of the mismatch angle between neighboring grains to an angle of approximately 20 degrees and then is essentially constant for all larger angles [16]. It is generally recognized that in a completely recrystallized solid, the driving force for grain growth lies in the surface energy of the grain boundaries [17]. The information on the previous section suggests that in a sample without the previous deformation, superplastic deformation starts with grainboundary sliding of individual grains, and later according to the second thermodynamic law during the superplastic deformation such grains it tends to grow (however such phenomena, it is almost controlled by different experimental techniques) and also grows a tendency to diminish the free energy per unit volume, $G_{P c s}(\bar{\theta})$, by diminishing the average mismatch angle, $\bar{\theta}$, between neighboring grains. This last phenomenon explains the tendency to grow the effective size on the gliding blocks formed by several grains, tendency described by the time evolution of the Gibbs energy per unit volume of the solid:

$$
G_{P c S}(\bar{\theta})=\left(4 N \pi r^{2}\right) \gamma(\bar{\theta})-T S,
$$

where $N$ is the number of grains per unit volume, $r$ is the mean grain radius, and $S$ is the configurationally entropy per unit volume due to the grains. In other words, if during a superplastic deformation test the number of grains remains constant, the only possibility to fulfill the second thermodynamic law for a system deforming superplastically far from thermodynamic equilibrium is

$$
\frac{d G_{P c s}(\bar{\theta})}{d t}=\left(4 N \pi r^{2}\right) \frac{d \gamma(\bar{\theta})}{d t} \leq 0 .
$$

Such condition implies that as far as the deformation test proceeds, the possibility of growing on the block size of sliding grains grows which is given by the time evolution of $\gamma(\theta)$,

$$
\frac{d \gamma(\bar{\theta})}{d t} \leq 0
$$

Many physical phenomena are described by the firstorder differential equations whose solution is an exponential decay which are common in solid state physics, medicine, biology and biophysics, geophysics, optics, engineering, chemistry, and electrochemistry [18]. In particular for polycrystalline materials, it is known that the general equations for the annealing of vacancies in metals containing impurities has shown that the decay curve is exponential [19], and also there is an exponential decay in nonlinear thermoelasticity [20], and in dislocation creep during a total unloading test, there is an exponential decay [21]; in all these cases, the system evolution occurs in a monotonic way and with an exponential decay of the free energy to its equilibrium value. Based on the previous data, it is possible to consider that during superplastic deformation the Gibbs free energy per unit volume $G_{P c s}(\bar{\theta})$ decays exponentially with time, in other words,

$$
\frac{d G_{P c s}(\bar{\theta})}{d t}=-\alpha e^{-\beta t} \leq 0,
$$

where $\alpha$ is a positive constant and $\beta \geq 0$ is the decay constant. By using (3), it is straightforward that

$$
\frac{d \gamma(\bar{\theta})}{d t}=-\left(\frac{\alpha}{4 N \pi r^{2}}\right) e^{-\beta t} .
$$

In such scheme, it is possible to suppose that the local or global cooperative grain-boundary sliding occurs by the gliding of blocks of grains which glide as an physical entity 
and has been formed by many neighboring grains with low grain boundaries energy states (low values of $\gamma(\bar{\theta})$ ). As the surface energy from grain boundaries provides the driving force for grain agglomeration which gives place to blocks of grains, then the simplest way to relate the change on the grain-boundary surface energy, $\gamma(\theta)$, and the rate of grain agglomeration is the following:

$$
\left(\frac{d r_{\text {Block }}}{d t}\right)\left(\frac{d \gamma(\bar{\theta})}{d t}\right)=C,
$$

where $C$ is assumed to be a constant and $\left(d r_{\text {Block }} / d t\right)$ is the rate of grain agglomeration which gives place to blocks of grains. Then,

$$
\left(\frac{d r_{\text {Block }}}{d t}\right)=C\left(\frac{4 N \pi r^{2}}{\alpha}\right) e^{+(\beta t)}
$$

With $\beta^{-1}=\tau$ and with $\tau$ as the exponential time constant, or grow time constant, it is clear that such thermodynamical process eventually gives place to the fracture of the sample under superplastic deformation.

2.1. Experimental Procedure. The material used for this experiment was a Cd-17.4\% Zn alloy. The alloy was prepared by melting cadmium and zinc in air furnace at $823^{\circ} \mathrm{K}$ and, then, poured into a copper ingot mold, and it was allowed to cool at room temperature. In order to avoid crack propagation during rolling due to the presence of dendrites, an annealing at $453^{\circ} \mathrm{K}$ was applied. The alloying element contents were measured by chemical method [22]. The material was laminated at room temperature at $4.6 \mathrm{~m} / \mathrm{s}$ with three rolling reduction steps $0.25 \mathrm{~cm}$ each, the plate obtained by lamination resulting in a grain size of $4.9 \mu \mathrm{m}$. After that, flat standard specimens for tensile tests parallel to the rolling direction were prepared with the following dimensions: $0.5 \mathrm{~cm}$ thick, $0.20 \mathrm{~cm}$ wide, and $1.6 \mathrm{~cm}$ of gage length. The samples were mechanical polished until reaching 0.5 micrometers diamond polishing paste. The tension tests, with an average constant crosshead velocity of $1.5 * 10^{-3} \mathrm{~cm} / \mathrm{s}$, were conducted at room temperature into a tension plate of a scanning electron microscope (SEM) model Jeol T20 which allows amplifications from 35 to 10,000 .

The new technique is a variation of one previously proposed [23-25] which provides a mesoscopic coordinate system inscribed on the surface of the tension test specimen. Such technique allows to establish in a repeatable way any angle relative to any axis of any coordinate system, or distances between grains, or to measure local or global true deformation in parallel or perpendicular directions relative to the tension axis. Here, such technique was adapted to measure grain area and perimeters of specific grains for a case where plastic deformation is so high that it is not possible to inscribe durable Vickers indentations on the surface of the deforming sample and, then, to take some particular defect of the sample as the origin of coordinated system is required.

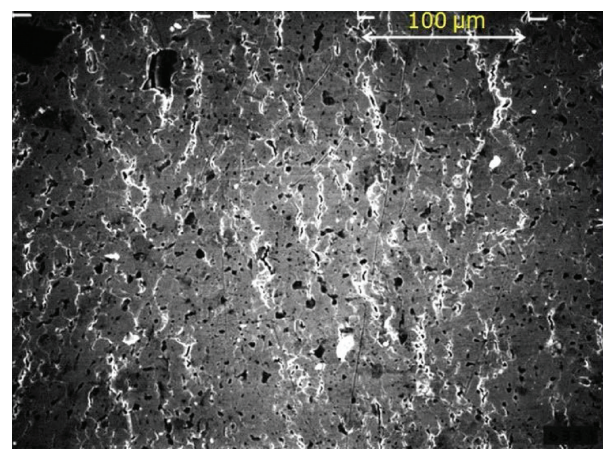

FIGURE 1: Scanning electron microscope micrograph for Cd-Zn eutectic alloy after $0.75 \mathrm{~mm}$ of elongation at room temperature, depicting GBS, which appears like bright lines.

\section{Experimental Data}

The local superficial deformation on different zones of the tension test sample was studied by using a coordinated reference system. During certain periods of time, the sample was deformed at the previously mentioned crosshead velocity; in each occasion after reaching some predetermined deformation test was interrupted to proceed to take photos of the superficial microstructure in different regions. The same procedure was repeated several times until fracture of the sample under tension occurs. The dispersion of experimental data was high, and only some data were presented to show the essential features of the corresponding phenomena. Among other parameters, the corresponding areas of emerging of grains or blocks of grains from the inner volume to the external surface of the sample, and their perimeters were measured for different times and deformations.

Figure 1 is a scanning electron microscope micrograph for Cd-Zn eutectic alloy after $0.75 \mathrm{~mm}$ of elongation at room temperature, depicting GBS, which appears like bright lines and corresponds to a typical zone in the surface of the deforming sample. During the experiments of sample deformation, two types of emerging populations of grains or blocks of grains from the inner volume to the external surface of the sample were found: emerging individual equiaxial grains and blocks of grains which arising from cavitation with form of cracks or fissures, as could be observed in Figure 2. These populations have different properties in their temporal evolution. In Figure 3, the rate of change of area of one emerging equiaxial grain is presented; it is clear that once the grains have emerged their changes in area are due to the interaction with neighboring grains during the plastic deformation of the sample. In Figure 4, the emergence of a block of grains appears arising from a fissure. The same zone appears in Figure 5 as a temporal sequence in a collage, where the kinetically process of the emerging of a block of grains to free surface could be appreciated; the quantitative data for such block appears in Figure 2. In Figure 6, some perimeter measurements are presented.

3.1. Analysis of Experimental Data. In order to obtain the scanning electron microscope micrograph for $\mathrm{Cd}-\mathrm{Zn}$ 


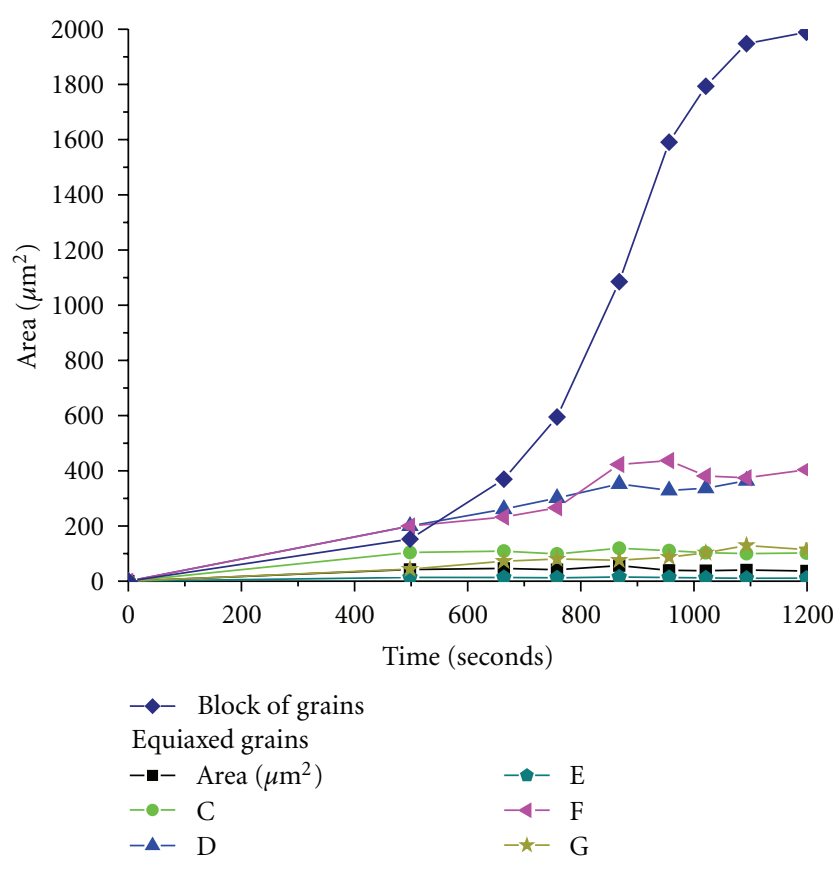

FIgURE 2: The area of grains from the inner volume emerging on the external surface of the sample during plastic deformation on a $\mathrm{Cd}-\mathrm{Zn}$ eutectic alloy as a function of time.

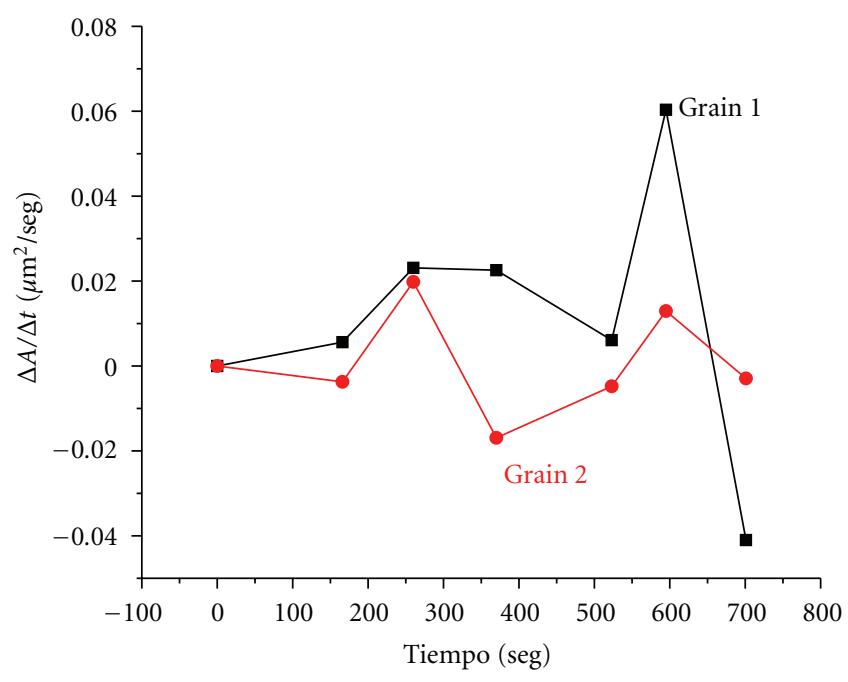

FIgURE 3: The rate of change of area of one emerging equiaxial grain emerging to the external surface from the inner volume as a function of time is presented for a Cd-Zn eutectic alloy deformed at room temperature.

eutectic alloy, it was necessary to stop the deformation for several minutes; this procedure may have led to possible changes in the microstructure observed, due to relaxation of internal stresses at the free surface. From Figure 3, it is immediate that during the plastic deformation the area of emerging equiaxed grains is changing with time by the interaction with their neighbors (above, below, and lateral neighboring grains). The positive change in area corresponds

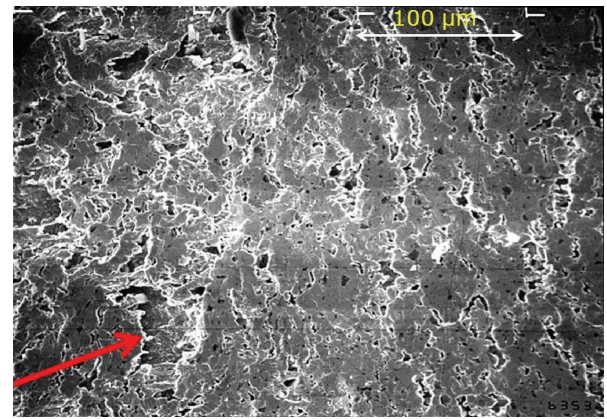

FIGURE 4: The emergence of a block of grains which travels from the inner volume and produces a fissure at the external surface.

to an emerging stage of some particular grains, and the negative area changes to a partial sinking process of the grain. Their shapes keep changing in the short term, but finally tend to reach a state of thermodynamic equilibrium (in particular mechanical equilibrium); please see Grain 2 in the figure. The materials emerging from the inside of cracks or fissures are block of grains formed by contiguous or neighboring grains which have common low-energy grain boundaries, that is, their common grain boundaries are strongly linked and slide like a unit, as can be seen from Figure 2, in which it is possible to see that the area of a totally emerged block of grains is about 26 times greater than the area of an average equiaxed grain. For our experimental data, showed in Figure 2, about material arising from the inside of cracks or fissures, it is clear that during the first 950 seconds an exponential growth on the area of the arising block of grain occurs, with a grow time constant of about $210.4 \mathrm{~s}$. Therefore, with our technique, it is possible to determinate some critical time (in our case $\tau_{\text {Crit }}=950 \mathrm{~s}$ ) for which the cracks or fissures can develop in two directions: (1) decreasing its speed, due to the total emerging of blocks of grains towards the surface or (2) continuing to grow exponentially as a crack that gives rise to fracture of the tension test sample under the action of the applied stress, because the block of grains partially under the free surface is not able to glide fast enough as required to fulfill the corresponding fissure. As shown in Figure 4, which corresponds to a block of grains that appears emerging from a fissure such fissure corresponds also to one of the areas depicted in Figure 2, for the block of grains where it is possible to see that the area of this block of grains as measured at the free surface of the sample under superplastic deformation tends to an equilibrium value. The corresponding kinetically processes indicated in Figure 5.

It is clear that the technique proposed and used here for Cd-Zn eutectic alloy could be applied to the study of the superplasticity phenomena as a complementary technique to the previously existing. In particular, such technique will be useful for the study of superplasticity in materials with nanocrystalline structures where, due to thermodynamical reasons, the kinetically evolution of the emerging of grains or blocks of grains from the inner volume to the free surface of superplastic material during deformation is expected to be higher than for the case of microcrystalline materials. 


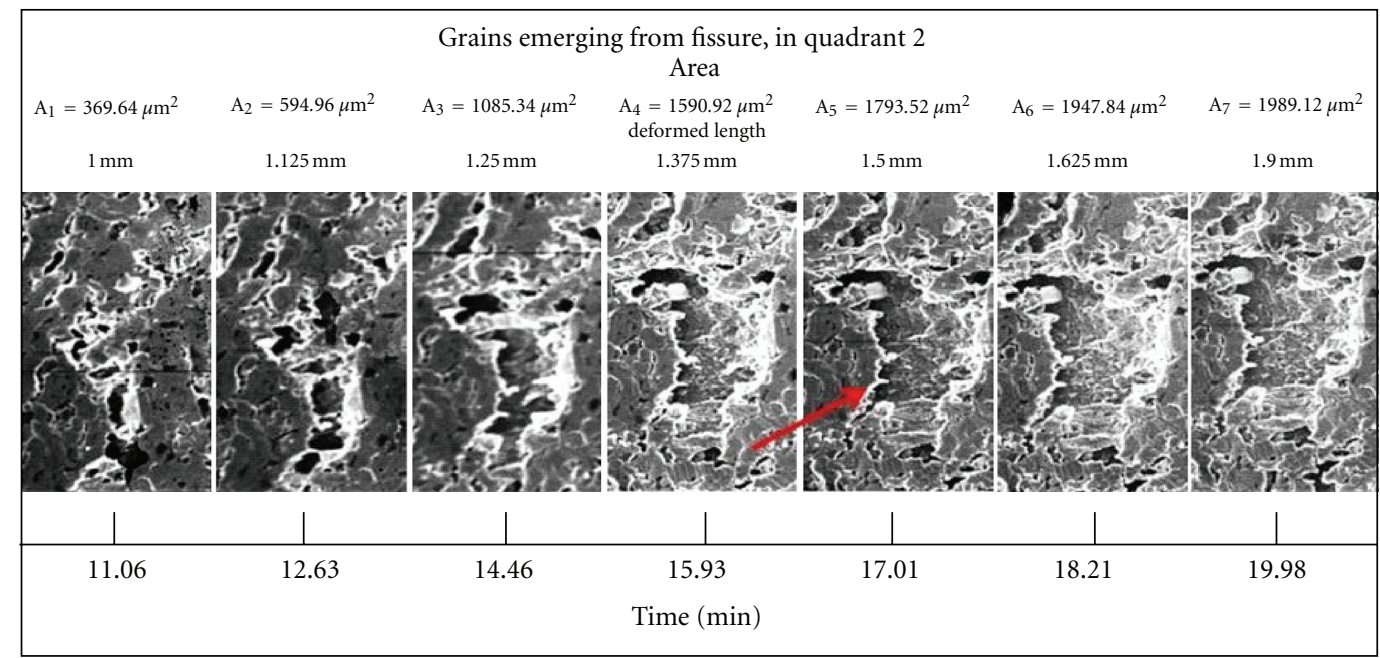

Figure 5: The same zone that appears in Figure 4 for the alloy Cd-Zn 17.4\% deformed at room temperature is presented here as a temporal sequence in a collage. The temporal emerging process of a block of grains to the external surface of the deforming sample could be appreciated; the plot of the quantitative data of area appears in Figure 2.

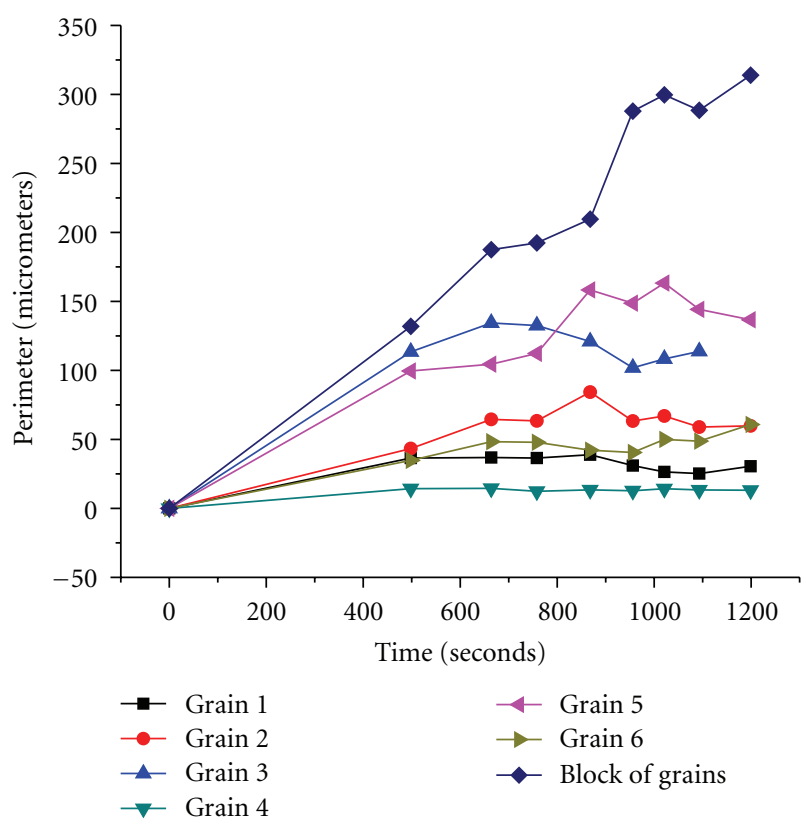

FIGURE 6: Some perimeter measurements for the alloy Cd-17.4\% $\mathrm{Zn}$ deformed at room temperature is presented.

\section{Discussion}

Our experimental results is to support the basis of a theory recently published [10], as well as in a more quantitative way the model here presented (based on the second law of thermodynamics) for the diminishing on the free energy per unit volume, $G_{P c s}(\bar{\theta})$, by diminishing the average mismatch angle, $\bar{\theta}$, between neighboring grains. And also, our results are in agreement with some experimental data for superplastic deformation in a $\mathrm{Zn}$-Al alloy which shows that cavities are initially reasonably spherical, but they become elongated when the sample pulls out to achieve the maximum superplastic elongation [11]; however, in our case, such cavities are mainly fulfilled with the grains or block of grains that merge to external surface from inferior layers. And obviously, for the case of superplastic deformation of the Cd-Zn eutectic alloy deformed at room temperature, we provide direct experimental evidence that GBS is the driving force for creep cavity nucleation, as deduced previously by an experimental correlation [13]. The technique presented here will be useful for the development of physical procedures, which allows to retarding the process of formation of low mismatch angle, $\bar{\theta}$, between neighboring grains; process which gives place to blocks of grains which dynamically behaves as units under the shear stress action. For materials with nanocrystalline structures, such processes are expected to be higher than for the case of microcrystalline materials.

\section{Conclusions}

(1) A new local measurement technique for the study of the kinetic processes of emerging of grains from the inner volume at the free surface of superplastic materials during deformation has been presented and used for the case of the Cd-Zn eutectic alloy deformed at room temperature.

(2) This technique can be used to evaluate the approximate time to fracture by fissure growth in samples under superplastic deformation.

(3) The technique presented here will be useful for the development of physical procedures, which allows to retarding the process of block by grains agglutination in materials with nanocrystalline structures, where by thermodynamical reasons, the process of formation of low mismatch angle, $\bar{\theta}$, between neighboring grains, which gives place to blocks of grains, is 
expected to be higher than for the case of microcrystalline materials.

(4) In principle, the present technique may be used to study the local surface forces which are acting over individual surface grains during the superplastic deformation.

\section{References}

[1] T. G. Langdon and J. Wadsworth, "Summary and topics of ICSAM-91," in Proceedings of the International Conference on Superplasticity in Advanced Materials, S. Hori, M. Tokizane, and N. Furishiro, Eds., pp. 847-852, The Japan Society for Research on Superplasticity, Osaka, Japan, 1991.

[2] M. F. Ashby and R. A. Verrall, "Diffusion-accommodated flow and superplasticity," Acta Metallurgica, vol. 21, no. 2, pp. 149 $163,1973$.

[3] M. G. Zelin, M. R. Dunlap, R. Rosen, and A. K. Mukherjee, "The direct observation of cooperative grain-boundary sliding and migration during superplastic deformation of lead-tin eutectic in shear," Journal of Applied Physics, vol. 74, no. 8, pp. 4972-4982, 1993.

[4] M. G. Zelin and M. V. Alexsandrova, "Cooperative grainboundary sliding during superplastic deformation," in Proceedings of the International Conference on Superplasticity in Advanced Materials, S. Hori, M. Tokizane, and N. Furishiro, Eds., pp. 95-101, The Japan Society for Research on Superplasticity, Osaka, Japan, 1991.

[5] V. V. Astanin, O. A. Kaibyshev, and S. N. Faizova, "Cooperative grain boundary sliding under superplastic flow," Scripta Metallurgica et Materiala, vol. 25, no. 12, pp. 2663-2668, 1991.

[6] H. S. . Yang, M. G. Zelin, R. Z. Valiev, and A. K. Mukherjee, "Strain induced morphological changes of $\alpha_{2}$ and $\beta$ phases in $\mathrm{Ti}_{3} \mathrm{Al}$ alloys during superplastic deformation," Scripta Metallurgica et Materialia, vol. 26, no. 11, pp. 1707-1712, 1992.

[7] M. G. Zelin and A. K. Mukherjee, "Study of cooperative grain boundary sliding by using macroscopic marker lines," Metallurgical and Materials Transactions A, vol. 26, no. 3, pp. 747-750, 1995.

[8] S. Krishnamurthy and A. H. Chokshi, "High temperature deformation and densification in alumina-yttria Composites," in Proceedings of the International Conference on Superplasticity in Advanced Materials (ICSAM '97), A. H. Chokshi, Ed., vol. 243-245 of Materials Science Forum, pp. 369-374, Trans Tech Publications, 1997.

[9] F. A. Mohamed, "Micrograin superplasticity: characteristics and utilization," Materials, vol. 4, no. 7, pp. 1194-1223, 2011.

[10] K. A. Padmanabhan, "Grain boundary sliding controlled flow and its relevance to superplasticity in metals, alloys, ceramics and intermetallics and strain-rate dependent flow in nanostructured materials," Journal of Materials Science, vol. 44, no. 9, pp. 2226-2238, 2009.

[11] M. Kawasaki and T. G. Langdon, "The development of internal cavitation in a superplastic zinc-aluminum alloy processed by ECAP," Journal of Materials Science, vol. 43, no. 23-24, pp. 7360-7365, 2008.

[12] V. N. Perevezentsev, V. V. Rybin, and V. N. Chuvil'deev, "The theory of structural superplasticity-IV. Cavitation during superplastic deformation," Acta Metallurgica Et Materialia, vol. 40, no. 5, pp. 915-924, 1992.
[13] C. R. Blanchard, R. A. Page, and S. Spooner, "The measurement of compressive creep deformation and damage mechanisms in a single-phase alumina part II correlation of creep cavitation and grain boundary sliding," Journal of Materials Science, vol. 33, no. 20, pp. 5049-5058, 1998.

[14] T. G. Langdon, "A unified approach to grain boundary sliding in creep and superplasticity," Acta Metallurgica et Materialia, vol. 42, no. 7, pp. 2437-2443, 1994.

[15] R. Z. Valiev, R. K. Islamgaliev, and N. F. Yunusova, "Superplasticity of nanostructured metallic materials obtained by methods of severe plastic deformation," Metal Science and Heat Treatment, vol. 48, no. 1-2, pp. 47-53, 2006.

[16] R. E. Reed-Hill, Physical Metallurgy Principles, Editorial D. Van Nostrand Co., Princeton, NJ, USA, 1968.

[17] R. E. Reed-Hill, Physical Metallurgy Principles, Editorial D. Van Nostrand Co., Princeton, NJ, USA, 1968.

[18] A. A. Istratov and O. F. Vyvenko, "Exponential analysis in physical phenomena," Review of Scientific Instruments, vol. 70, no. 2, pp. 1233-1257, 1999.

[19] A. C. Damask and G. J. Dienes, "Theory of vacancy annealing in impure metals," Physical Review, vol. 120, no. 1, pp. 99-104, 1960.

[20] J. E. Muñoz Rivera and R. Kamei Barreto, "Existence and exponential decay in nonlinear thermoelasticity," Nonlinear Analysis: Theory, Methods and Applications, vol. 31, no. 1-2, pp. 149-162, 1998.

[21] J. A. Montemayor-Aldrete and M. López de Haro, "A statistical mechanical approach to dislocation creep and the total unloading test on Al-11wt Zn alloy," in Advances in Science, Technology and Applications of Zn-Al Alloys, G. Torres Villaseñor, Y. H. Zhu, and C. Piña, Eds., pp. 115-1120, U.N.A.M., México, Mexico, 1994.

[22] J. E. Moreno and G. Torres-Villaseñor, "In situ observations of the superplastic deformation of Cd-Zn eutectic alloy," in Advanced Materials' 93, III/B: Composites, Grain Boundaries and Nanophase Materials, M. Sakai et al., Ed., vol. 16 of Transactions of the Materials Research Society of Japan, pp. 1023-1026, 1994.

[23] J. D. Muñoz-Andrade, A. Mendoza-Allende, G. TorresVillaseñor, and J. A. Montemayor-Aldrete, "Mapping of the granular flow during superplastic deformation of microsample of $\mathrm{Zn}-20.2 \% \mathrm{Al}-1.8 \% \mathrm{Cu}$ alloy at room temperature," Journal of Materials Science, vol. 36, no. 3, pp. 795-799, 2001.

[24] J. A. Montemayor-Aldrete, J. D. Muñoz-Andrade, G. TorresVillaseñor, and A. Mendoza Allende, "Superplasticity: fundamental problems to be solved," Recent Research Developments in Metallurgical \& Materials Sciences, vol. 5, pp. 11-13, 2001.

[25] J. D. Muñoz-Andrade, A. Mendoza-Allende, G. TorresVillaseñor, and J. A. Montemayor-Aldrete, "Cooperative grain boundary sliding at room temperature of a $\mathrm{Zn}-20.2 \% \mathrm{Al}-$ $1.8 \% \mathrm{Cu}$ superplastic alloy," Materials Science Forum, vol. 357359, pp. 551-558, 2001. 

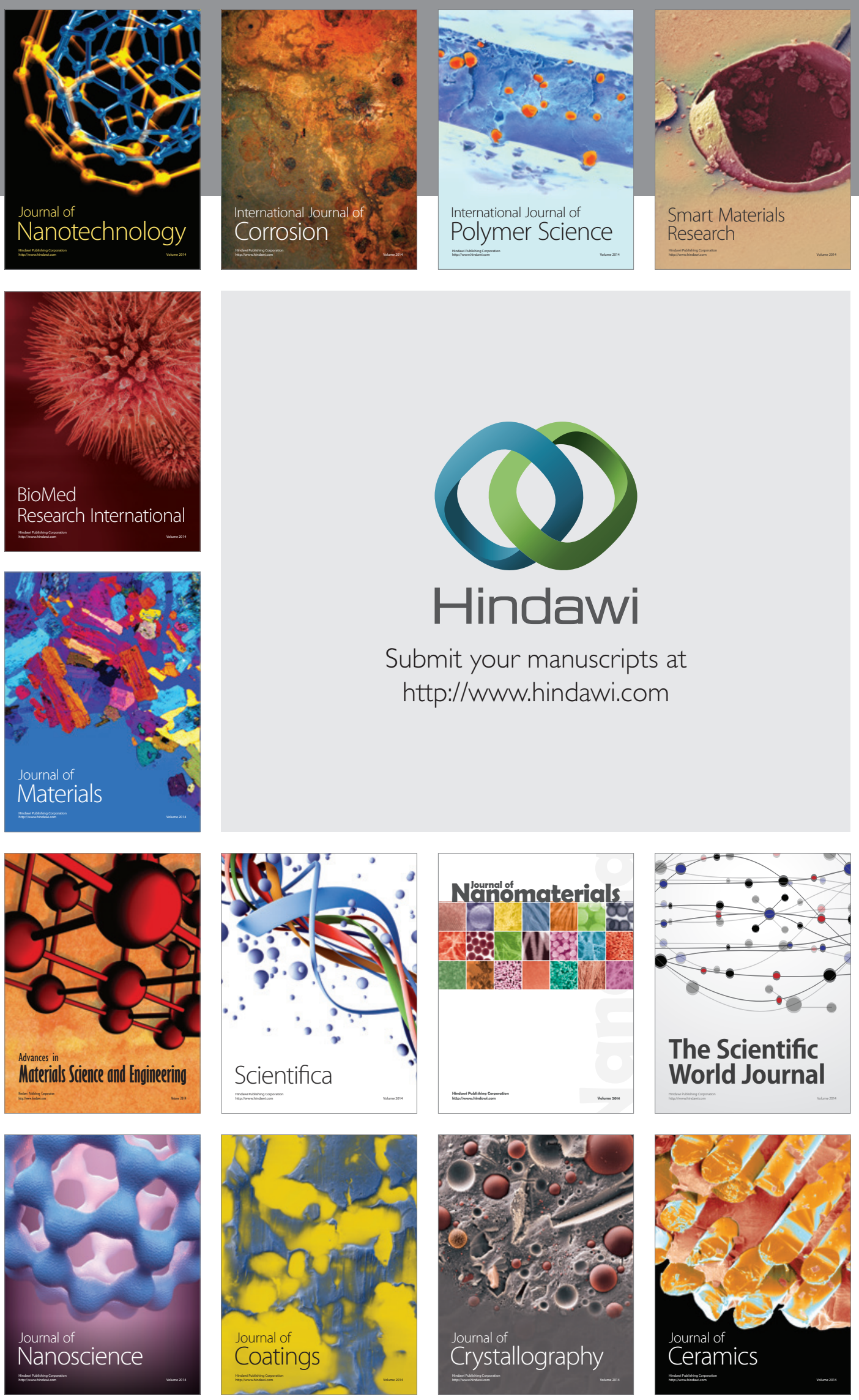

The Scientific World Journal

Submit your manuscripts at

http://www.hindawi.com

\section{World Journal}

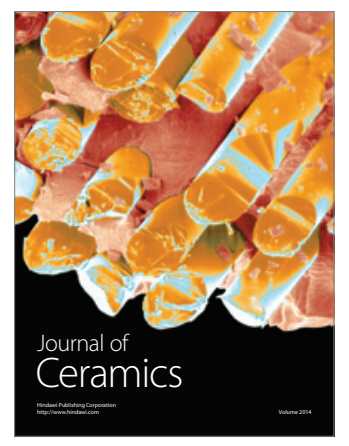

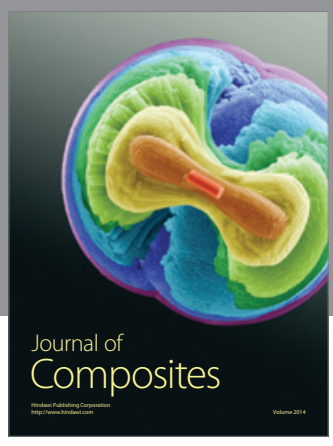
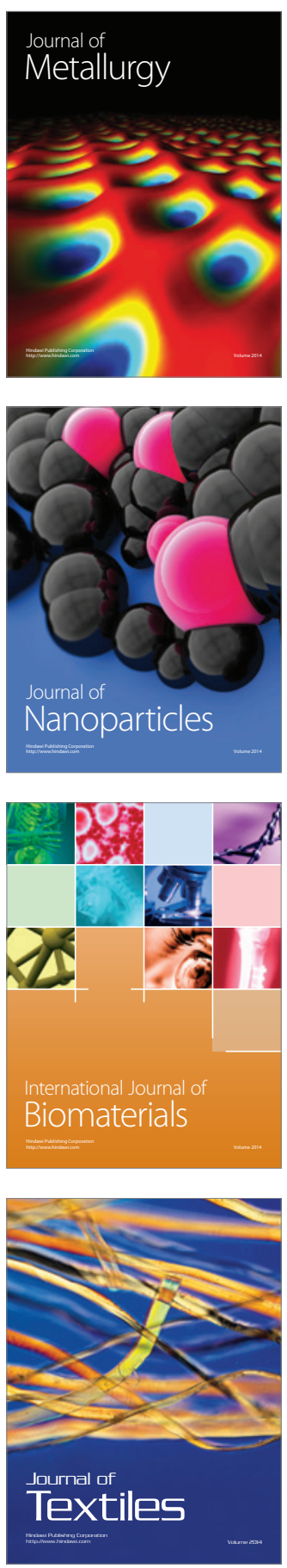